\title{
Experimental investigation of the influence of freestream turbulence on an anti-vortex film cooling hole
}

\author{
Stephen A. Hayes, Andrew C. Nix ${ }^{*}$, Christopher M. Nestor, David T. Billups, Shane M. Haught \\ Department of Mechanical and Aerospace Engineering, West Virginia University, 271 ESB Annex, Morgantown, WV 26506-6106
}

\section{A R T I C LE INF O}

\section{Article history:}

Received 00 December 00

Received in revised form 00 January 00

Accepted 00 February 00

\section{Keywords:}

Gas Turbine

Film Cooling

Heat Transfer

Infrared Thermography

Anti-Vortex Hole

\begin{abstract}
A B S T R A C T
Film cooling is used in gas turbines to thermally protect combustor and turbine hot section components by creating a layer of relatively cooler air to insulate the components from the hot freestream gases. This relatively cooler air is taken from upstream in the compressor section at a loss to the engine efficiency and therefore must be used as effectively as possible. A novel anti-vortex hole (AVH) geometry has been investigated experimentally through a transient infrared thermography technique to study the film cooling effectiveness and heat transfer coefficient by varying blowing ratio and freestream turbulence intensity. The AVH geometry is designed with two secondary holes stemming from a main cooling hole that attempts to diffuse the coolant jet and mitigate the vorticity produced by conventional straight holes. The AVH geometry data collected showed improved cooling performance at low freestream turbulence intensities compared to conventional straight holes. Three turbulence intensities of $1,7.5$, and $11.7 \%$ were investigated for the AVH geometry at blowing ratios of $0.5,1.0,1.5$, and 2.0 for a total of twelve different conditions. Results showed that higher freestream turbulence conditions were beneficial to the performance of the AVH. Increasing the blowing ratio at all turbulence levels also improved film cooling effectiveness, both span-averaged and on the centerline. The highest performing case was at a turbulence intensity of $7.5 \%$ and a blowing ratio of 2.0 . The $11.7 \%$ cases outperformed the $1 \%$ cases, but it appears that at the $11.7 \%$ cases the higher freestream turbulence reduces the performance of the secondary holes compared to the $7.5 \%$ case. Increasing the blowing ratio and turbulence intensity will result in a higher heat transfer coefficient, which must be taken into account for future designs and overall reduction of heat load.
\end{abstract}

(C) 2016 Hosting by Elsevier B.V. All rights reserved.

\section{Introduction}

One primary way to increase the efficiency and power output of a gas turbine engine is to raise the combustor exit temperature, and therefore, the turbine inlet temperature. The melting point of the materials in the combustor and turbine sections are often exceeded and the components can and will fail due to thermal loading if not cooled. As a result, relatively cooler air from the compressor section upstream must be extracted and used to cool the components in the turbine section. Cooling of components occurs through internal cooling passages or externally via film cooling holes. Film cooling is an external cooling method in which the coolant is ejected through discrete holes in the airfoil surface creating a layer of insulation over the surface, protecting it from the hot freestream gas in the region directly downstream of the holes. For areas of low curvature on turbine airfoils, cooling of these regions can be modeled by flat plate studies. Multiple types of cooling hole geometries have been studied including, but not limited to the conventional straight hole, shaped holes, and multi-hole concepts. Kadotani and Goldstein [1,2] determined that freestream turbulence

* Corresponding author. Tel.: +1 304293 0801; fax: +1 3042936689

E-mail address: andrew.nix@mail.wvu.edu

Peer review under responsibility of Xxxxx.

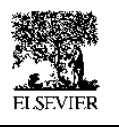

\section{Hosting by Elsevier}

xxxx-xxxx/\$ - see front matter (C) 2013 xxxxxxxx. Hosting by Elsevier B.V. All rights reserved.

http://dx.doi.org/10.1016/j.rgo.2013.10.012 
intensity, blowing ratio (BR), and the ratios of turbulence length scale to cooling hole diameter and length scale to boundary layer thickness were of greatest importance when studying the variation of film cooling effectiveness. Blowing ratio is defined in equation 1 below where $\rho_{c}$ is the coolant density, $\rho_{\infty}$ is the freestream density, $U_{c}$ is the coolant velocity exiting the holes, $\mathrm{U}_{\infty}$ is the freestream velocity.

$$
\mathrm{BR}=\rho c U c \rho \infty \bigcup_{\infty}
$$

Han and Mehendale [3] found for a single row of conventional straight holes that a blowing ratio between 0.5 and 0.7 was best for coverage of film cooling. Two significant issues with standard hole configurations are the lift off that occurs as the coolant jet exits the hole at higher blowing ratios and the formation of vortices that entrain the hot freestream gas along the airfoil surface. Haven et al. [4] described and illustrated in detail, this phenomenon known as a counter-rotating vortex (CRV) pair as seen below in Fig. 1

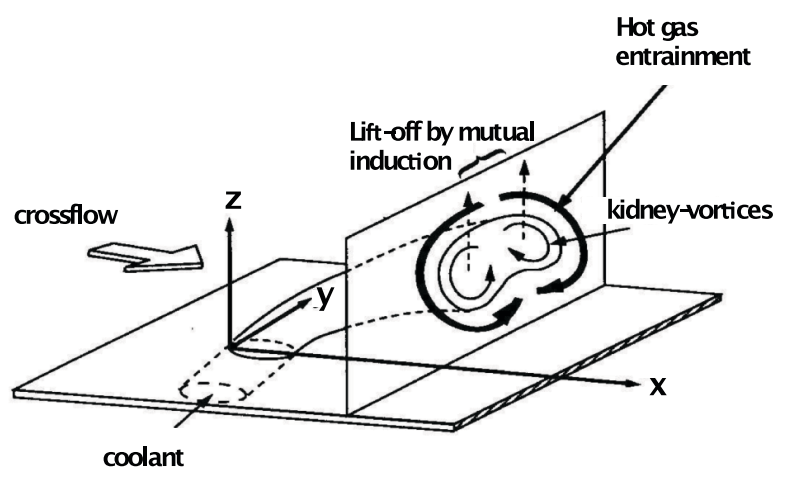

Fig. 1. Counter-rotating vortex pair and jet lift-off from Haven et al. [4]

The combination of the CRV pair and blowing ratios in gas turbine engines more commonly being 1.5 and above led to research into mitigating the CRV pair at higher blowing ratios with the use of different cooling hole geometries. The main idea behind shaped and multi-hole designs is derived from the need to decrease the jet velocity out of the holes by increasing the hole exit area relative to the hole entrance area therefore reducing the jet lift off. Bunker [5] presented a review of shaped holes that included a laidback (allowing expansion downstream along the surface) and a fan shaped hole (allowing lateral expansion) as well as combinations of the two.

The focus of the current work is to experimentally investigate the effectiveness of a multi-hole cooling concept, the anti-vortex hole (AVH) geometry, and to analyze the effects of freestream turbulence on cooling performance at varying blowing ratios. The AVH configuration, shown in Fig. 2, consists of a main cooling hole with two sister holes that branch out from the main hole. As shown in the figure, the hole geometry is defined by several angles and spacings. Details of the actual geometry tested in the current study are shown later in Table 1. The AVH geometry is an attempt to reduce both lift off and vortex generation. The benefits of the AVH geometry were realized through CFD simulations prior to this investigation $[6,7]$ and for low freestream turbulence intensities through experimentation [8]. This geometry was selected because it was the optimal performing configuration of this geometry for low freestream turbulence [8]. Previous studies to measure and evaluate the effects of freestream turbulence intensity on this AVH geometry were performed solely computationally $[9,10,11,12]$. These studies showed that the AVH geometry has improved cooling performance as turbulence intensity increases. The current paper will report on experimental measurements of the effect of turbulence intensity on cooling performance of the AVH geometry.

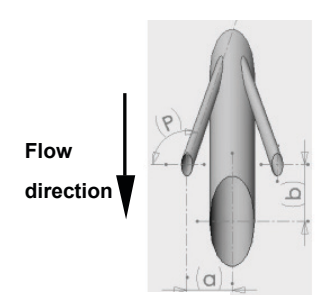

Top View

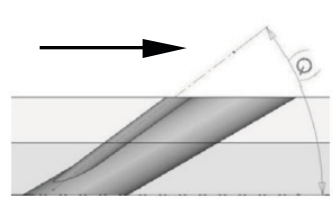

Side View

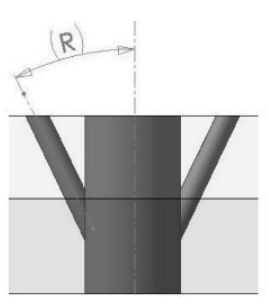

Front View

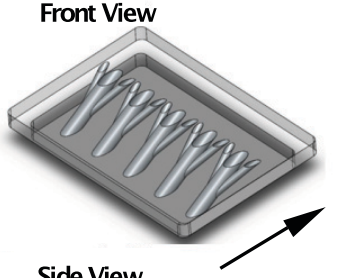

Side View
Fig. 2. Three View Drawing of Anti-Vortex Hole [8]

\begin{tabular}{|c|c|}
\hline \multicolumn{2}{|l|}{ Nomenclature } \\
\hline $\mathrm{BR}$ & \\
\hline $\mathrm{C}_{\mathrm{p}}$ & $\begin{array}{l}\text { blowing ratio or mass flux ratio }(\rho \mathrm{V})_{\mathrm{c}} /(\rho \mathrm{V})_{\infty} \\
\text { specific heat at constant pressure }\end{array}$ \\
\hline $\mathrm{D}$ & main film cooling hole diameter \\
\hline $\mathrm{h}$ & heat transfer coefficient \\
\hline $\mathrm{k}$ & thermal conductivity \\
\hline $\mathrm{p}$ & hole pitch \\
\hline $\operatorname{Re}$ & Reynolds number \\
\hline $\mathrm{t}$ & time \\
\hline $\mathrm{T}$ & temperature \\
\hline $\mathrm{Tu}$ & turbulence intensity \\
\hline $\mathrm{U}$ & velocity \\
\hline $\mathrm{x}$ & streamwise direction from hole leading edge \\
\hline $\mathrm{z}$ & span wise direction from the hole centerline \\
\hline Greek & \\
\hline$\alpha$ & thermal diffusivity, coolant injection angle \\
\hline$\delta$ & boundary layer thickness \\
\hline$\eta$ & film cooling effectiveness \\
\hline
\end{tabular}




\begin{tabular}{|ll|}
\hline$\eta_{\text {aw }}$ & adiabatic film cooling effectiveness \\
$\rho$ & density \\
Subscripts & \\
aw & adiabatic wall \\
c & coolant conditions \\
i & coordinate direction (parallel with x), pixel \\
RVU & number \\
freestream inlet conditions \\
f
\end{tabular}

\section{Experimental Facility}

The experimental facility at West Virginia University (WVU) shown in Fig. 3 and Fig. 4 is an open loop tunnel that has been designed and fabricated to non-dimensionally simulate the aerothermal environment experienced by the first stage of a gas turbine engine as a flat plate representation. The motor at full load is capable of $3625 \mathrm{RPM}(380 \mathrm{rad} / \mathrm{s})$ resulting in tunnel freestream velocities of approximately $25 \mathrm{~m} / \mathrm{s}(82 \mathrm{ft} / \mathrm{s})$. The cross-sectional area ratio through the diffuser is $2: 1$ and is designed with an expansion angle as small as possible in an attempt to prevent boundary layer separation [13]. The mesh heaters are capable of a step temperature increase of approximately $10^{\circ} \mathrm{C}\left(18^{\circ} \mathrm{F}\right)$ above ambient at the current freestream flow Reynolds number (Re) based on hole diameter (D) of approximately 10,000. These resistance heaters are made from 304 stainless steel wire mesh screens connected in series so as to reduce the current requirements on the welder used to provide current. The mesh is a $200 \mathrm{x}$ 200 wire per square inch arrangement with wire diameter of 0.0016 inches and a spacing of 0.0034 inches between wires resulting in an open area of $46 \%$ for each screen. The three current options for turbulence intensities are $1 \%$ (no grid), and $7.5 \%$, and $11.7 \%$ using passive style grids, measured at the leading edge of the coolant holes. Turbulence intensity was measured using a Dantec Dynamics 54T42 MiniCTA hotwire anemometer survey in the streamwise direction downstream of the grids to insure that heat transfer measurements were performed beyond the region of decaying turbulence. The hotwire has a frequency bandwidth up to $10 \mathrm{kHz}$. The test section is $15.24 \mathrm{~cm}$ (6 in.) tall by $25.4 \mathrm{~cm}$ (10 in.) wide and is $91.55 \mathrm{~cm}$ (36 in.) long. The non-dimensionalized boundary layer height based on cooling hole diameter $(\delta / D)$ is 0.5 at the cooling hole leading edge. In the coolant air loop, shown in Fig. 5, pressurized house air passes through a Meriam 50MC2 laminar flow element (LFE) to meter the coolant flow, and is then routed to a copper tube bank submerged in a dry ice and ethylene glycol bath reducing the air temperature by $9^{\circ} \mathrm{C}$ before feeding this air to the cooling hole plate plenum. This arrangement achieves a freestream to coolant temperature ratio $T_{\infty} / T_{c}$ of 1.06, which is lower than that for engine conditions. A valve in-line with the cooling loop allowed for adjustment of the blowing ratio yielding the four blowing ratios used in this study. Activating film cooling into the heated mainstream flow creates a transient condition used to determine film effectiveness and heat transfer coefficient simultaneously, as described later. The test section base allows for interchangeability of geometries through the use of plates, which eliminates the need to remanufacture the test section base. The base of the test section is made of one inch thick machinable black ABS plastic due to its low thermal conductivity, approximating an adiabatic condition. The current facility was benchmarked with the use of the conventional straight hole geometry as ample data was available in open literature for comparison to validate the facility. Five Omega K-type thermocouples were also placed on the surface in regions of varying temperature with one being located in the hottest and coldest locations to ensure interpolation of temperature rather than extrapolation when used in conjunction with a FLIR A655sc infrared (IR) camera for full test surface temperature measurement. 


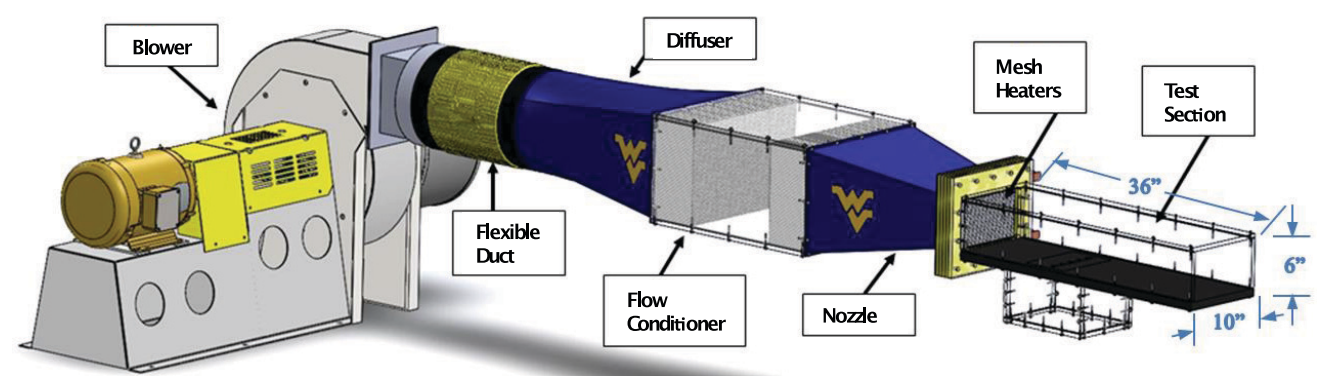

Fig. 3. Drawings of Wind Tunnel Facility Freestream Loop

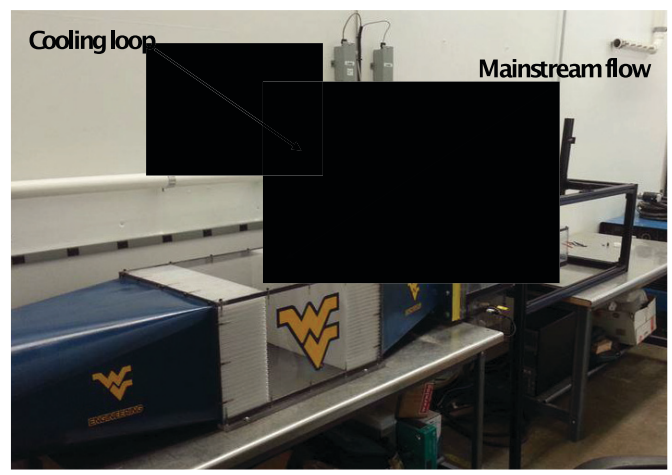

Fig. 4. Drawings of Wind Tunnel Facility Freestream Loop

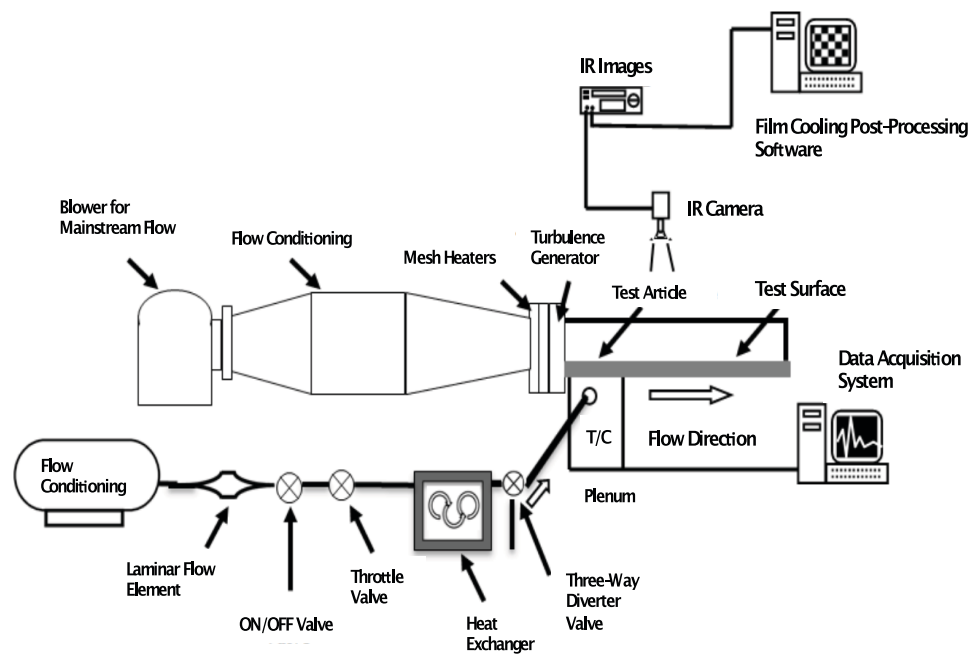

Fig. 5. Wind Tunnel Facility

\section{Methodology}

\subsection{Transient heat transfer}

The transient case of a flow passing over a semi-infinite solid flat plat is known as a two-temperature problem where the freestream temperature and wall temperature are the only drivers for heat transfer. In principal the surface can be thought of as infinite in all but one direction (z), which is into the wall $[14,15]$. This allows one to assume that when a sudden change occurs at the surface a transient one-dimensional conduction into the solid transpires as a result. The governing equation for one-dimensional unsteady heat conduction is:

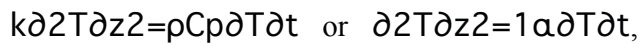

$$
\text { where } a=k \rho C p
$$

where $\rho$ is the density, $\mathrm{k}$ is the thermal conductivity, $\mathrm{C}_{\mathrm{p}}$ is the specific heat and $\alpha$ is the thermal diffusivity of the ABS plastic and are known properties 
of the surface material. Furthermore, $\mathrm{T}$ is the temperature and $\mathrm{t}$ is the time. The boundary and initial conditions for this case are:

$$
\begin{array}{r}
-k \partial \mathrm{T} \partial \mathrm{z}=\mathrm{hT} \infty-\mathrm{T} 0, \mathrm{t} \\
\mathrm{Tl} \mathrm{z}=\infty=\mathrm{Ti} \\
\mathrm{Tlt}=0=\mathrm{Ti}
\end{array}
$$

Here $T_{i}$ is the initial uniform wall temperature, $T_{\infty}$ is the freestream flow, $\mathrm{T}$ is the varying temperature of the surface material with time, and $\mathrm{h}$ is the convective heat transfer. The assumption that the plate is a semi-infinite solid means that the heat transfer into the top of the plate cannot be experienced by the bottom of the plate during the experiment. This can be measured by the penetration depth which is given in equation (6):

\section{Penetration Depth $=$ at}

Here $\alpha\left(10.76 \times 10^{-8} \mathrm{~m}^{2} / \mathrm{s}\right)$ is the thermal diffusivity of the ABS plastic and $\mathrm{t}$ is the time of the transient event, which was 60 seconds. Solving this equation yields a penetration depth of approximately $2.5 \mathrm{~mm}(0.1 \mathrm{in}$.). The plate is $6.35 \mathrm{~mm}(0.25 \mathrm{in}$.) thick so this assumption is adequately satisfied. Solving equation (2) with the outlined conditions yields:

\section{Tw-TiTs-Ti=1 -exph2atk2erfchatk=1-erfcxhatk}

Here $\mathrm{h}$ is the unknown heat transfer coefficient, again $(\alpha)$ is the thermal diffusivity of the ABS plastic, $k$ is the thermal conductivity of the ABS plastic, and $t$ is the duration of time from the initiation of the transient test. Equation (7) above is furthermore applicable because a true step temperature change is imposed upon the freestream flow. $T_{w}$ is measured through the transient test with an infrared camera and calibrated with multiple thermocouples located along the test surface as described in section 2 .

\subsection{Transient Film Cooling}

As stated previously, equation (7) is for a two-temperature problem, but film cooling introduces a three-temperature problem where the freestream temperature, wall temperature, and injected coolant temperature drive the heat transfer [16,17]. Equation (7) must be adjusted to incorporate the film temperature from the coolant and freestream interacting and results in:

\section{Tw-TiT $\infty-\mathrm{Ti}_{1}=1-$ erfcxhatk}

where the complementary error function is:

$$
\text { erfcx }=1-e r f x
$$

and the scaled complementary error function is:

$$
\operatorname{erfcxx}=\exp x 2 \star \operatorname{erfx}
$$

Equation (8) has two unknown values which are the film temperature and heat transfer coefficient, but of greater interest than the film temperature is another variable which is based on the film temperature and is known as film cooling effectiveness. The highest possible value of film cooling effectiveness is one when the film temperature is equal to the coolant temperature and the lowest value of zero occurs when the film temperature is equal to the freestream temperature. Film cooling effectiveness is a nondimensional term and can be shown as:

$$
\eta=T f-T_{\infty} T c-T_{\infty} \text { or } \quad \eta a w=T_{\infty}-T_{a w} T_{\infty}-T_{c}
$$

where $T_{a w}$ is the adiabatic wall temperature condition that is used for low temperature testing (relative to engine conditions) and does not account for any heat loads due to radiation. Rearranging to solve for $\mathrm{T}_{\mathrm{f}}$ :

$$
T f=\eta * T c-T \infty+T \infty=\eta * T c+1-\eta * T \infty
$$

Taking equation (8) and solving for $T_{w}-T_{i}$ and replacing $T_{f}$ with equation (12) yields:

$\mathrm{Tw}-\mathrm{Ti}=1-$ erfchatk $\star \eta * T c+1-\eta * \mathrm{~T}_{\infty}-\mathrm{Ti}$

with the two desired unknowns being the heat transfer coefficient (h) and the film cooling effectiveness $(\eta)$.

Ekkad [16] proposed utilizing infrared thermography in a method previously used by Vendula and Metzger [17]. Vendula and Metzger used thermochromatic liquid crystal paint wherein two different time instances from a single transient test could be used to simultaneously solve a system of equations with two unknowns ( $h$ and $T_{f}$ ). These two equations occur at the two separate time instances, $t_{1}$ and $t_{2}$ with wall temperatures $T_{w 1}$ and $T_{w 2}$, respectively, and can be shown as:

Tw1-TiTf-Ti=1-erfcxha $t$ k

\section{Tw2-TiTf-Ti=1-erfcxhat $2 \mathrm{k}$}

This method adequately solves for the correct values of the film temperature and heat transfer coefficient, and therefore the film cooling effectiveness from equation (11), but during early iterations of data reduction it was found that the resulting values were not consistent if the time between selected frames was varied. To resolve this issue a regression analysis was applied to compute reliable and consistent results using multiple frames.

\subsection{Regression Analysis}

To reiterate, it is desired to solve for $\mathrm{h}$ and $\eta$ which can be done by solving simultaneously the aforementioned two equations (14 and 15) with these two unknowns, but it is desirable to minimize the uncertainty in these solutions as much as possible. The test surface is painted flat black to ensure high emissivity and is viewed through a stretched polyethylene window. The window serves two critical functions in the test: preventing mass flow loss from the wind tunnel test section while providing high transmissivity of the IR signature of the test surface. For each test, data is collected at $25 \mathrm{~Hz}$ in the IR camera software code, and four frames are selected for the initial wall temperature just before the transient test begins and ten frames are selected 6 seconds apart through the 60 seconds of the transient temperature change event. To extract the data from the software and process into desired format, numerous steps must be performed to reduce error. Five surface 
thermocouples with known locations on the test surface are matched with their respective pixel on the recorded IR image. Due to the step temperature change of the freestream, the polyethylene window undergoes a temperature change as well and results in a non-uniformity in transmissive losses. For each thermocouple at a single time instant, the external optics temperature is changed to match each thermocouple individually then the average of the five temperatures is used for the external optics temperature (window temperature) and that frame is then output for further data reduction. Once this is done for all 14 frames, the thermocouple temperatures and radiance values are exported into a MATLAB ${ }^{\circledR}$ program where a best fit is applied to the thermocouple values to camera radiance values. A least squares solution is applied and the new corrected temperatures are applied to all 307,200 pixels in the image reducing the error from camera and thermocouple readings. The four frames used for the initial surface temperature are averaged together in an attempt to account for any pixels that may have fluctuated due to camera errors, as bad pixel readouts may occur.

In an attempt to further reduce uncertainty, an iterative non-linear leastsquare regression analysis technique was used to solve for $\mathrm{h}$ and $\eta$ for a single experiment as presented by $\mathrm{Lu}$ [14]. From the previous steps, the known time at each frame $t_{I R}$ when a specific camera measured value $T_{I R}$ appears during transience can be shown as:

\section{Twh, $\eta t=t I R=T I R$}

Where this equation applies separately to each individual pixel of each frame which can be expressed mathematically as:

\section{Twh, $\eta \mathrm{t}=\mathrm{tIR}-\mathrm{TIR}=0 \quad \forall \mathrm{i}=1$ to $\mathrm{N}$}

where $\mathrm{i}$ is the given pixel and $\mathrm{N}$ is the number of frames being used. When more than two frames are being used to solve for $h$ and $\eta$ for these equations with the least amount of error, the following error function is used:

$i=1 \mathrm{~N}[\mathrm{Twh}, \mathrm{\eta t}=\mathrm{t} I R i-\mathrm{T} I R i] 2=\mathrm{e}(\mathrm{h}, \eta) \geq 0$

Taking equation (18) and substituting into equation (13) allows for an iterative method to solve for $\mathrm{h}$ and $\eta$ by minimizing the right hand side (the error). Using the "fminsearch" function in $\mathrm{MATLAB}^{\circledR}$ and controlling the maximum number of function evaluations $(150,000)$ and maximum number of iterations $(100,000)$ per pixel and entering initial guesses and bounds for $h$ and $\eta$, these values could be computed between 20 and 120 minutes depending upon the number of evaluations and iterations needed to reach the prescribed convergence tolerances. This method reduces the error that is present in the infrared measurements and relaxes the dependence upon the initial wall temperature by applying additional time instances in the data processing and error reduction.

\subsection{Experimental Uncertainty}

Experimental uncertainty was estimated based on the method outlined by Moffat [18] by introducing a bias uncertainty for each of the measured quantities and calculating a precision uncertainty based on test statistics. The bias uncertainties were determined based on manufacture specifications and were perturbed in the regression code sequentially. The resulting values of $h$ and $\eta$ were determined at each pixel for the applied error. The values which were perturbed were $\mathrm{T}_{\infty}\left( \pm 0.5^{\circ} \mathrm{C}\right), \mathrm{T}_{\mathrm{c}}\left( \pm 0.5^{\circ} \mathrm{C}\right), \mathrm{T}_{\mathrm{i}}\left( \pm 0.125^{\circ} \mathrm{C}\right), \mathrm{T}_{\mathrm{w}}\left( \pm 0.125^{\circ} \mathrm{C}\right)$, $\mathrm{t}( \pm 0.125 \mathrm{sec})$ and $\mathrm{k}( \pm 0.01 \mathrm{~W} / \mathrm{m}-\mathrm{K})$. The temperature biases for $\mathrm{T}_{\mathrm{w}}$ and $\mathrm{T}_{\mathrm{i}}$ were each lower than the $T_{\infty}$ and $T_{c}$ biases because a curve fit was applied between the five surface thermocouples. Further, the assumption was made that not all thermocouples could have a $+0.5^{\circ} \mathrm{C}$ reading or $-0.5^{\circ} \mathrm{C}$ at the same time. Once all values had been perturbed and the codes had been executed, the positive perturbation and negative perturbation of each adjusted measurement were taken and their absolute values were averaged. These values are then used to find the root-mean-square (RMS) value of the bias uncertainty.

The second factor is the precision uncertainty and is based on a student$\mathrm{t}$ distribution based on the number of tests performed and test points collected. Finally the total uncertainty can be determined from the bias and precision uncertainties shown in equation (19) for the film cooling effectiveness:

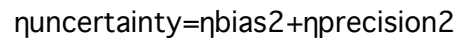

The final uncertainties determined were \pm 0.05 for centerline film cooling effectiveness and \pm 0.024 for span-averaged film cooling effectiveness. The heat transfer coefficient uncertainties were $\pm 28 \%$ for the centerline heat transfer coefficient and $\pm 33 \%$ for the span-averaged heat transfer coefficient. The high uncertainty in the convective heat transfer coefficient is likely due to the fact that the values had to be computationally solved rather than directly measured experimentally with heat flux sensors or foil heaters, but the comparative convective heat transfer values between test conditions are more important in determining trends between tests rather than the absolute value or high uncertainty.

\section{Results}

The current study was conducted to assess the performance of an antivortex hole (AVH) geometry illustrated in Fig. 1. The geometric parameters of the specific AVH design are presented in Table 1. This design was the optimized design in a study done by Dhungel et al. $[8,15]$ and was selected for this reason. The chief concern with the AVH geometry is the effectiveness that the secondary holes will have in counteracting the downwash of the main hole coolant jet counter rotating vortices (CRV). Table 2 outlines the test matrix of the experiments performed at blowing ratios of $0.5,1.0,1.5$, and 2.0 with turbulence intensities of $1,7.5$, and $11.7 \%$ for a total of 12 cases. Each experiment was conducted four times to ensure accuracy and precision of the results. Results are presented for film cooling effectiveness, convective heat transfer coefficient and net heat flux reduction using contour plots and streamwise variations of centerline and span averaged data.

\section{Table 1}

Geometric Parameters of Current AVH Geometry

\begin{tabular}{cc}
\hline Dmain $(\mathrm{cm})$ & 1.27 \\
\hline Dsecondary $(\mathrm{cm})$ & 0.63 \\
\hline $\mathrm{a}(\mathrm{cm})$ & 1.42 \\
\hline $\mathrm{b}(\mathrm{cm})$ & -0.56 \\
\hline $\mathrm{P}\left({ }^{\circ}\right)$ & 105.67 \\
\hline $\mathrm{Q}\left(^{\circ}\right)$ & 26.41 \\
\hline $\mathrm{R}\left({ }^{\circ}\right)$ & 27.91 \\
\hline
\end{tabular}

Table 2

Experimental Test Matrix

\begin{tabular}{ccc}
\hline Experiment & Blowing Ratio (BR) & $\begin{array}{c}\text { Turbulence } \\
\text { Intensity (Tu) }\end{array}$ \\
\hline
\end{tabular}




\begin{tabular}{ccc}
\hline Experiment & Blowing Ratio (BR) & $\begin{array}{c}\text { Turbulence } \\
\text { Intensity (Tu) }\end{array}$ \\
\hline $\mathrm{A}$ & 0.500 & $1.00 \%$ \\
\hline $\mathrm{B}$ & 1.00 & $1.00 \%$ \\
\hline $\mathrm{C}$ & 1.50 & $1.00 \%$ \\
\hline $\mathrm{D}$ & 2.00 & $1.00 \%$ \\
\hline $\mathrm{E}$ & 0.50 & $7.50 \%$ \\
\hline $\mathrm{F}$ & 1.00 & $7.50 \%$ \\
\hline $\mathrm{G}$ & 1.50 & $7.50 \%$ \\
\hline $\mathrm{H}$ & 2.00 & $7.50 \%$ \\
\hline $\mathrm{I}$ & 0.50 & $11.7 \%$ \\
\hline $\mathrm{J}$ & 1.00 & $11.7 \%$ \\
\hline $\mathrm{K}$ & 1.50 & $11.7 \%$ \\
\hline $\mathrm{L}$ & 2.00 & $11.7 \%$ \\
\hline
\end{tabular}

\subsection{Film Cooling Effectiveness}

\subsubsection{Experimental Validation}

In order to validate the experimental data collected and presented herein, a sample of results at low turbulence $(\mathrm{Tu}=1 \%)$ and a $\mathrm{BR}$ of 1.0 were compared on a span-averaged basis with another experimental study (Dhungel et al. [8]) and with a current CFD study being conducted at WVU for a conventional straight hole geometry. The results are shown below in Fig. 6. In general, the WVU experimental data agrees well with both the previous experimental data and CFD. Some differences magnitude and location of jet lift-off (although lift-off is better illustrated with centerline data) are present.

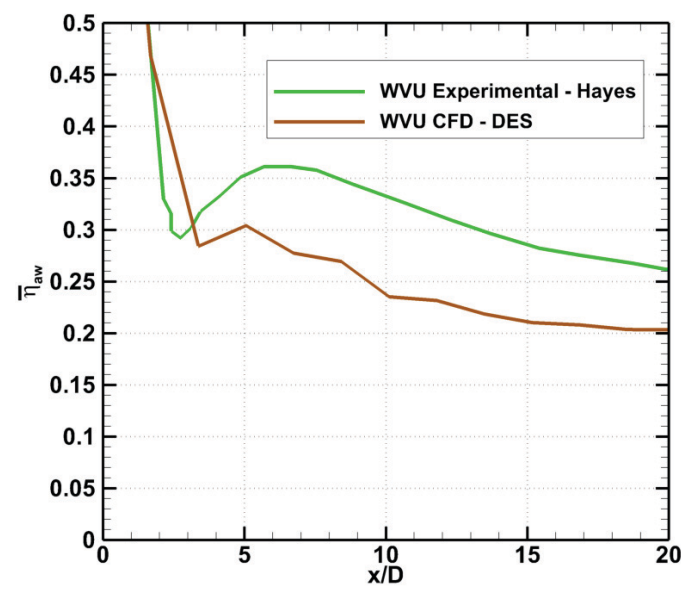

Fig. 7. Comparison of Experimental Data with CFD-DES (AVH hole, span averaged, $\mathrm{Tu}=1 \%$ and $\mathrm{BR}=1.0$ )

\subsubsection{Contour Plots of Effectiveness}

Contour plots allow for a general view of overall cooling effectiveness while showing specific locations of increased or diminished cooling which would not be as easily discernable through streamwise plots of the variation of span-averaged and centerline cooling effectiveness. Fig. 8a and Fig. 8b display the film cooling effectiveness contour plots of the experiments performed for a non-dimensionalized stream wise distance of 27 main cooling hole diameters $(\mathrm{x} / \mathrm{D})$ and spanwise distance from -1.5 to 1.5 (the pitch to diameter of the main hole, $\mathrm{p} / \mathrm{D}$, is 3.0). For the low turbulence case of $1 \%$ it is clearly visible through the use of the contour plots that the film

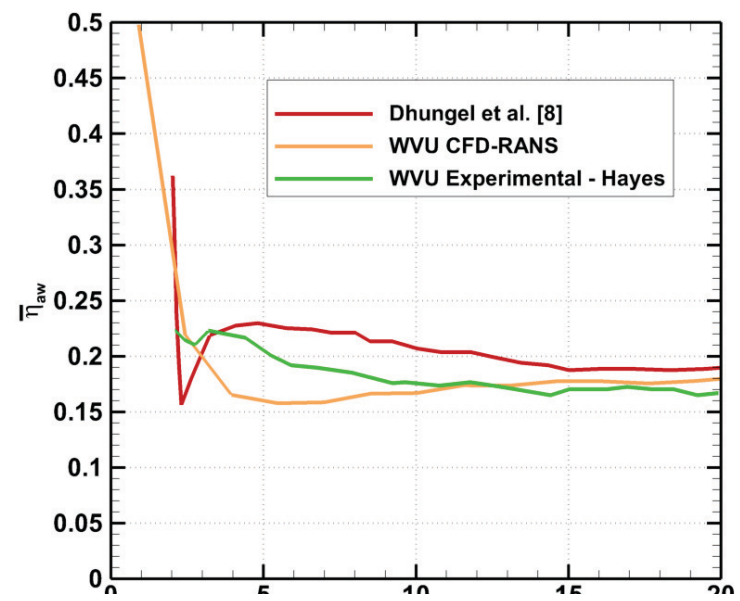

Fig. 6. Comparison of Experimental Data with Previous Experiments and $\mathrm{CFD}$ (conventional straight hole, span averaged, $\mathrm{Tu}=1 \%$ and $\mathrm{BR}=1.0$ )

Fig. 7 presents a comparison of span-averaged film cooling effectiveness at low turbulence $(\mathrm{Tu}=1 \%)$ and a $\mathrm{BR}$ of 1.0 for the $\mathrm{AVH}$ geometry at for the current study to detached eddy simulation (DES) data from a current study being conducted at West Virginia University (WVU). Again, this data shows very good agreement in the streamwise variation of cooling with some some difference in magnitude after approximately $\mathrm{x} / \mathrm{D}$ of 5.0 .

cooling effectiveness is enhanced with an increase in blowing ratio not only in the lateral direction, but the streamwise direction as well. Distinct cooling is prevalent out of the two secondary holes on the midline between the main holes and are actually the regions of highest effectiveness.

At higher freestream turbulence, the lateral spreading of the coolant jets is improved over that in the low turbulence cases with it appearing that the best performing coverage occurs for the $7.5 \%$ cases for blowing ratios of 1.5 and 2.0 respectively. With experimental results, it is demonstrated that while freestream turbulence increases cooling effectiveness, there is a point at which further increasing the turbulence intensity results in decreasing performance.

\subsubsection{Span-Averaged, Centerline and Area-Averaged Effectiveness}

Fig. 9 through Fig. 111 display the span-averaged and centerline film cooling effectiveness values at each of the turbulence intensities for all four blowing ratios. Span-averaged film cooling effectiveness is the prevailing gauge in determining the overall performance when studying cooling hole geometries, while the centerline plots allow for determining the manifestation of jet lift off. The span-averaged and centerline film cooling effectiveness at turbulence intensities of 1 and $7.5 \%$ are the highest at a blowing ratio of 2.0. The span-averaged effectiveness at a blowing ratio of 0.5 is lowest in all cases except for the 2-3 hole diameters directly behind the trailing edge of the cooling hole. The centerline effectiveness improves with increased blowing ratio for the 1 and $7.5 \%$ turbulence cases and is the opposite of that experienced by conventional straight hole designs [19,20]. Improvement of the centerline effectiveness is a highly desired result for real applications because it shows jet lift off reduction. 


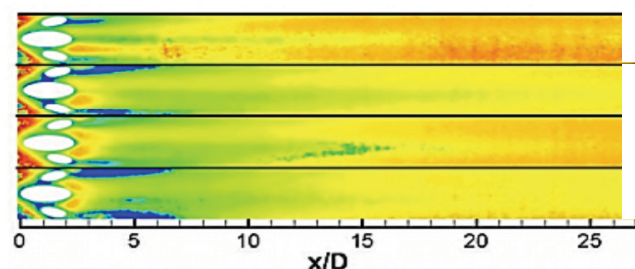

$\mathrm{BR}=0.5 \mathrm{Tu}=1 \%$

$\mathrm{BR}=1.0 \mathrm{Tu}=1 \%$

$\mathrm{BR}=1.5 \mathrm{Tu}=1 \%$

$\mathrm{BR}=20 \mathrm{Tu}=1 \%$

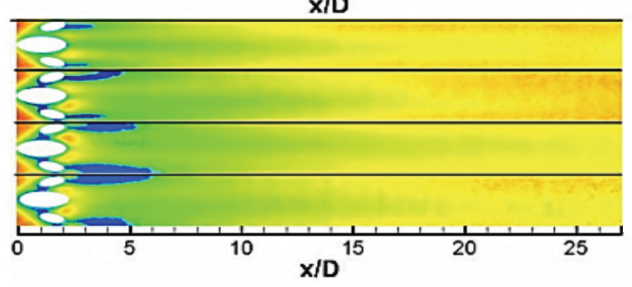

$\mathrm{BR}=0.5 \mathrm{Tu}=7.5 \%$

$\mathrm{BR}=1.0 \mathrm{Tu}=7.5 \%$

$B R=1.5 \mathrm{Tu}=7.5 \%$

$B R=20 T u=7.5 \%$

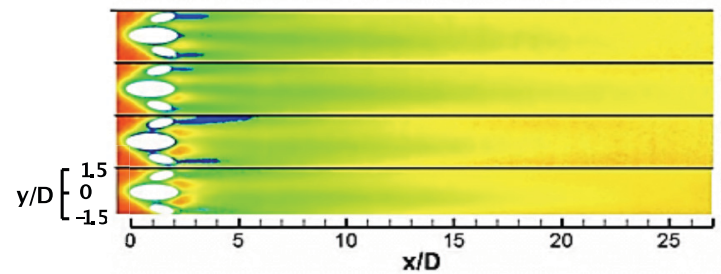

$B R=0.5 \mathrm{Tu}=11.7 \%$

$B R=1.0 T u=11.7 \%$

$B R=1.5 \mathrm{Tu}=11.7 \%$

$B R=20 \mathrm{Tu}=11.7 \%$

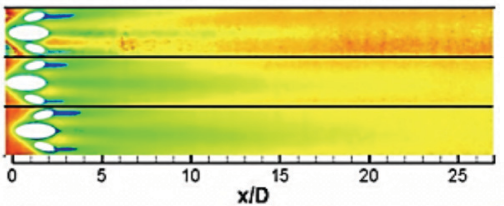

$\mathrm{BR}=0.5 \mathrm{Tu}=1 \%$

$B R=0.5 \mathrm{Tu}=7.5 \%$

$B R=0.5 \mathrm{Tu}=11.7 \%$

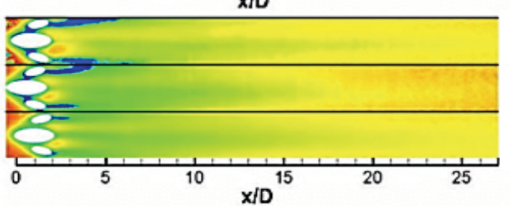

$B R=1.0 T u=1 \%$

$\mathrm{BR}=1.0 \mathrm{Tu}=7.5 \%$

$B R=1.0 T u=11.7 \%$

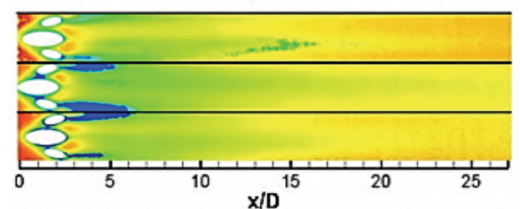

$B R=1.5 \mathrm{Tu}=1 \%$

$B R=1.5 \mathrm{Tu}=7.5 \%$

$B R=1.5 \mathrm{Tu}=11.7 \%$

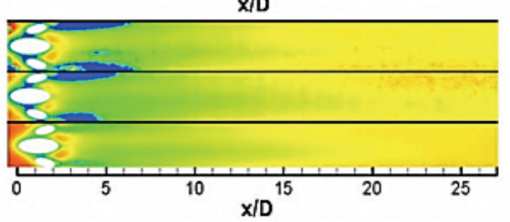

$B R=20 T u=1 \%$

$B R=20 T u=7.5 \%$

$B R=20 T u=11.7 \%$

(a)

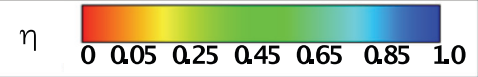

(b)

Fig. 8. (a) Film Cooling Effectiveness Contour Plots of Varying Blowing Ratios at a Constant Turbulence Intensity

(b) Film Cooling Effectiveness Contour Plots of Varying Turbulence Intensity at a Constant Blowing Ratio 


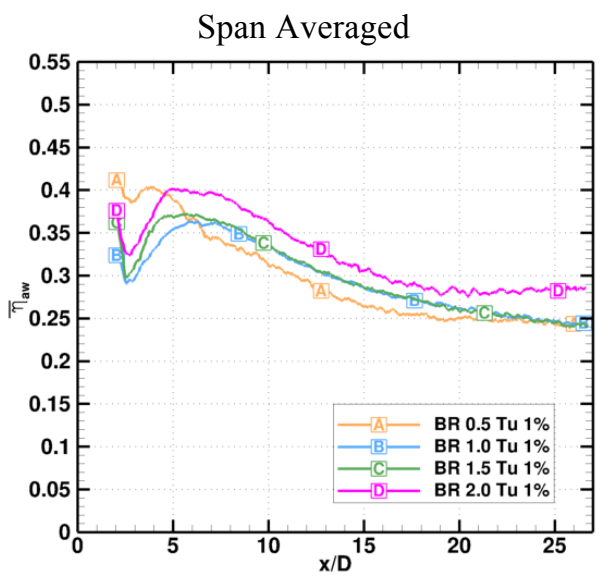

(a)

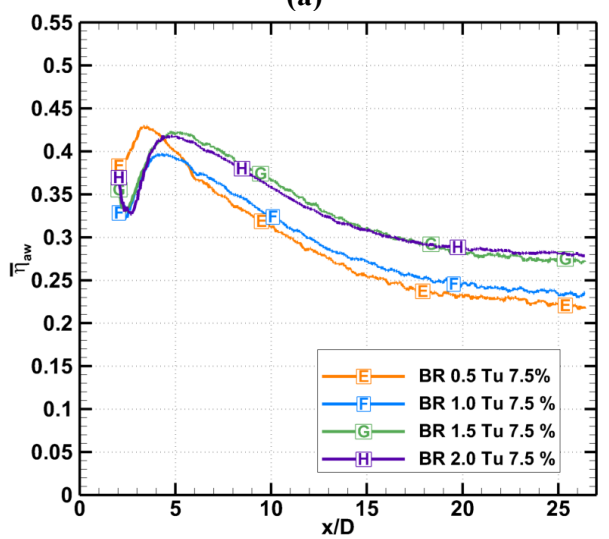

(b)

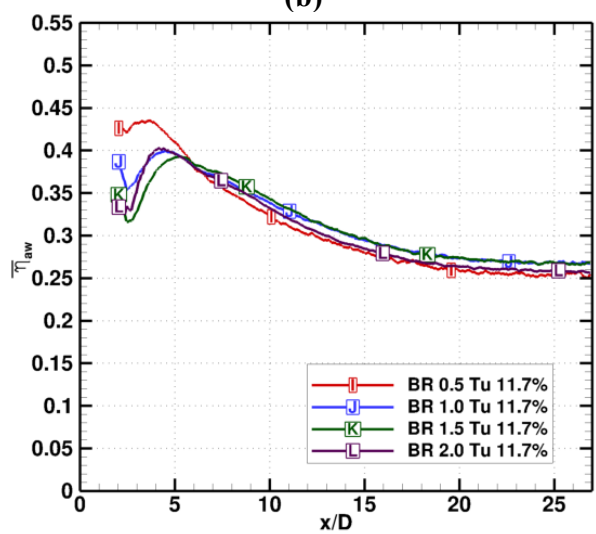

(c)

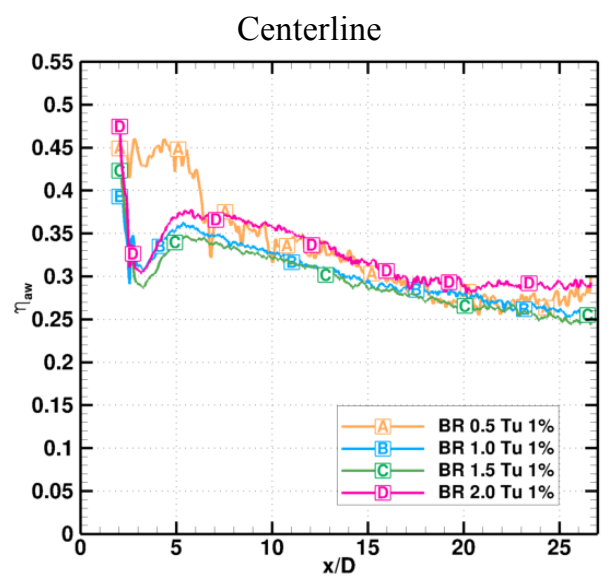

(d)

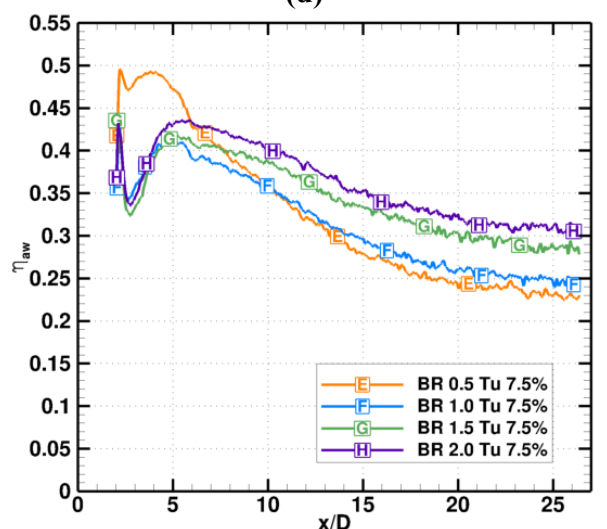

(e)

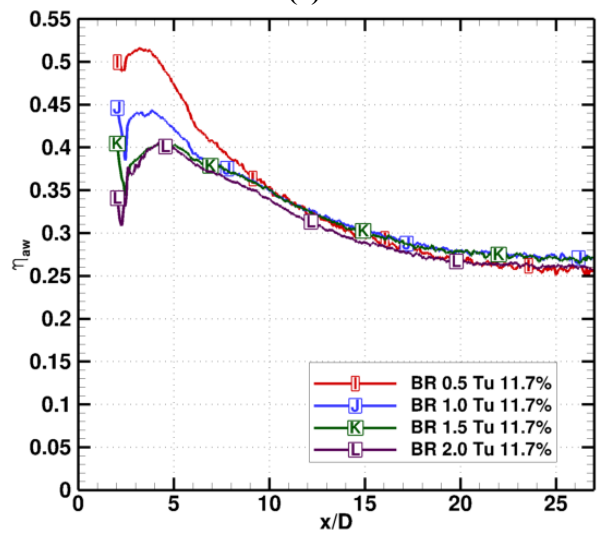

(f)

Fig. 9. (a-c) Span-averaged Film Cooling Effectiveness at Varying Blowing Ratios at a Constant Turbulence Intensity (d-f) Centerline Film Cooling Effectiveness Contour Plots at Varying Blowing Ratios at a Constant Turbulence Intensity

The highest effectiveness occurs at the turbulence intensity of $7.5 \%$ and a blowing ratio of 2.0 beyond 5 hole diameters as can be seen in Fig. 9e. This is possible because at this turbulence intensity the coolant jets spread more laterally than the $1 \%$ case and the secondary hole vortices are capable of still counteracting the main hole CRV pair.

At a turbulence level of $11.7 \%$ the span-averaged and centerline effectiveness trend to similar effectiveness past an $\mathrm{x} / \mathrm{D}$ location of 5 . At a turbulence intensity of $11.7 \%$ the span-averaged effectiveness is lower than the $7.5 \%$ case, most noticeably at blowing ratios of 1.5 and 2.0 which is most likely due to the enhanced mixing of the freestream with the coolant and the CRV pairs of the secondary holes being weakened making them less capable of cancelling out the CRV of the main hole. Fig. 11 displays the centerline data and at higher blowing ratios jet lift off is still prevalent, but is still the highest at a turbulence intensity of $7.5 \%$. The span-averaged and centerline film cooling effectiveness reaches a maximum at approximately 5 hole diameters downstream as presented in Fig. 9.

As noted in previous studies discussed herein, the primary mechanism for the increasing cooling performance in the presence of high freestream turbulence with the AVH hole is the decrease in jet momentum due to the 
side holes and subsequent increase in lateral spreading as turbulence intensity increases. This was shown computationally in Repko et al. [12]. 


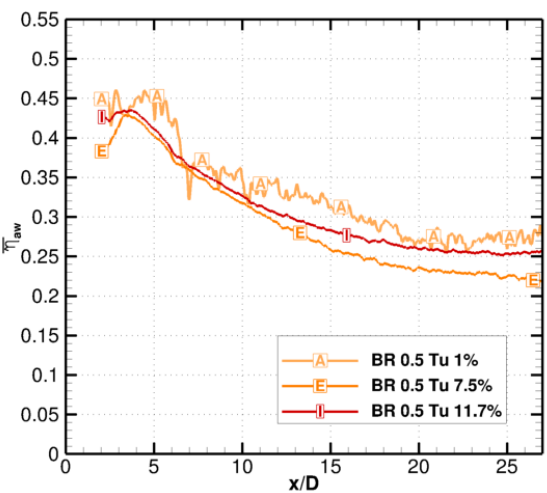

(a)

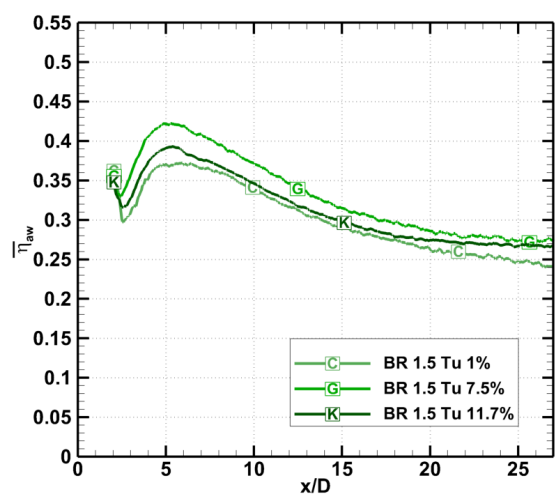

(c)

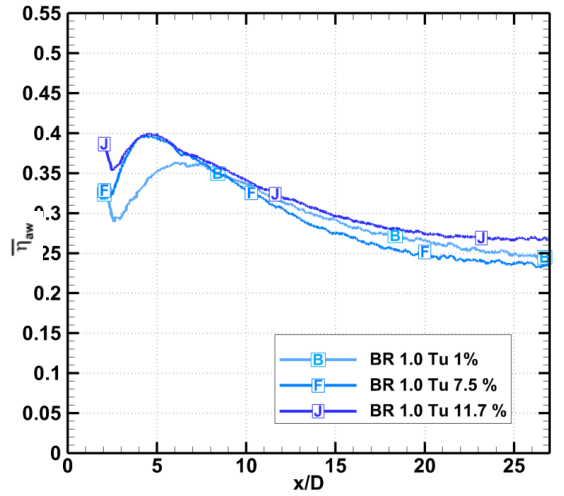

(b)

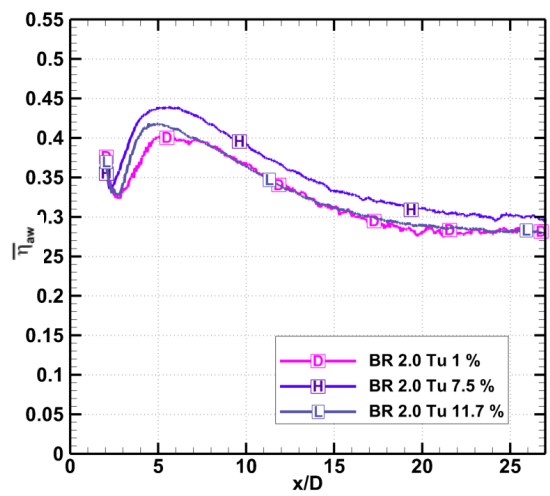

(d)

Fig. 10. Span-averaged Film Cooling Effectiveness at Varying Turbulence Intensity and Constant Blowing Ratio

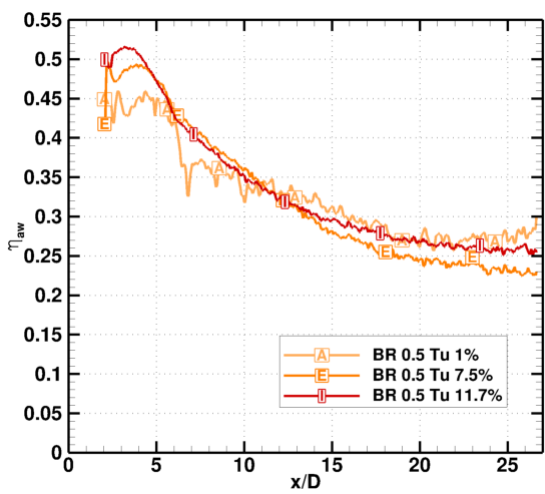

(a)

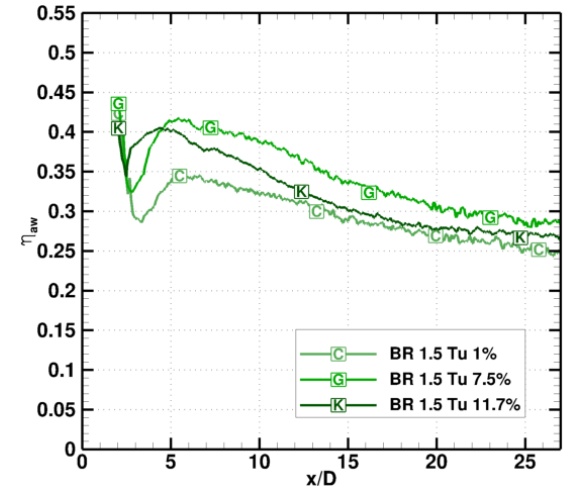

(c)

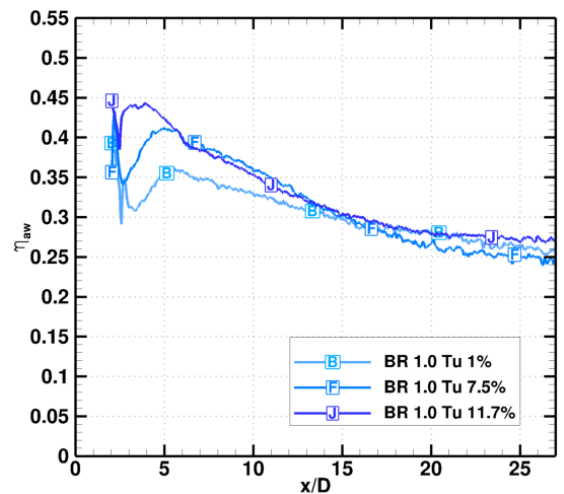

(b)

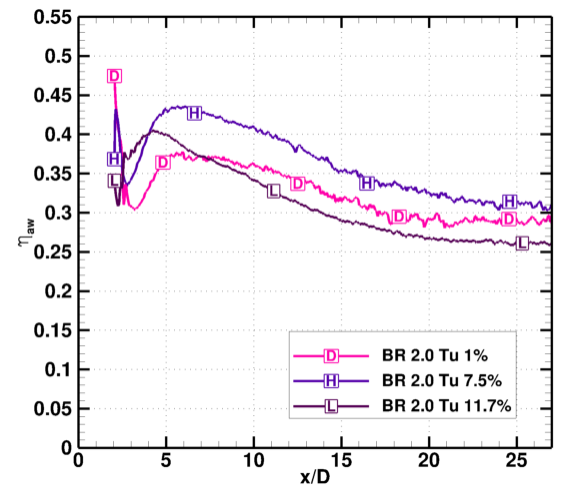

(d)

Fig. 11. Centerline Film Cooling Effectiveness at Varving Turbulence Intensity and Constant Blowing Ratio 
The area-averaged film cooling effectiveness is presented in Table $\mathbf{3}$ for each blowing ratio and turbulence intensity. At each of the turbulence intensities the general trend is that as the blowing ratio is increased, the film cooling effectiveness is also increased. The two best cases were at a blowing ratio of 2.0 and a blowing ratio of 1.5 for the $7.5 \%$ turbulence cases. The two highest turbulence cases generally outperformed the same blowing ratio cases for the low turbulence intensity which demonstrates that high freestream turbulences actually augments area-averaged film cooling effectiveness, with the effectiveness increasing with turbulence intensity, but not demonstrating a decrease with continued in increase in intensity as observed with the span-average and centerline effectiveness.

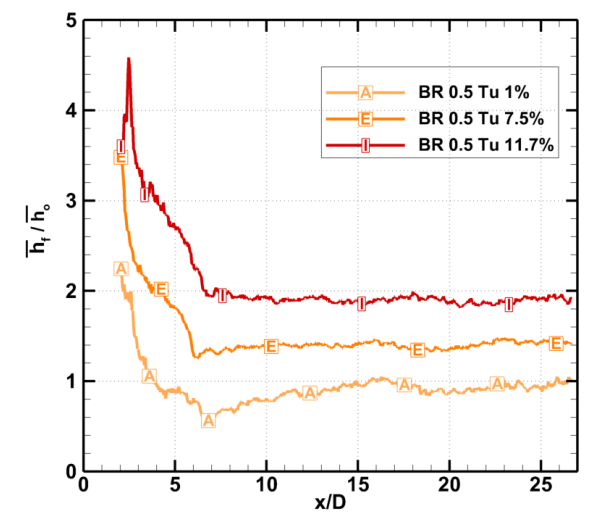

(a)

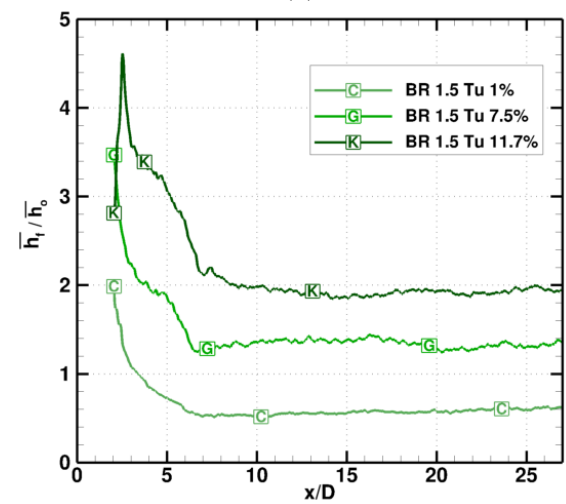

(c)
Table 3

Area- averaged Film Cooling Effectiveness

\begin{tabular}{cccc}
\hline Case & Tu 1.00\% & Tu 7.50\% & Tu 11.7\% \\
\hline BR 0.5 & 0.328 & 0.288 & 0.308 \\
\hline BR 1.0 & 0.296 & 0.294 & 0.314 \\
\hline BR 1.5 & 0.300 & 0.330 & 0.312 \\
\hline BR 2.0 & 0.325 & 0.351 & 0.329
\end{tabular}

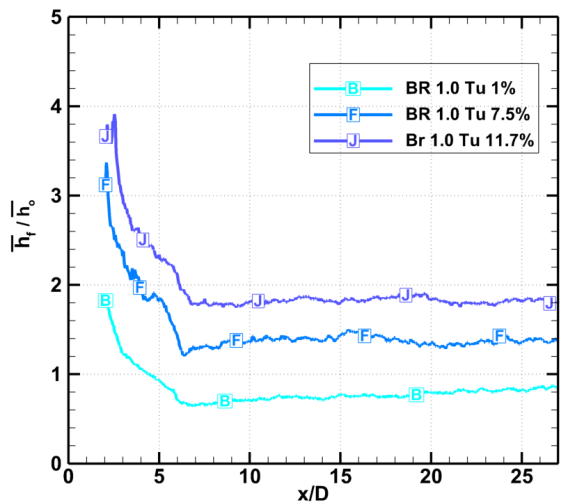

(b)

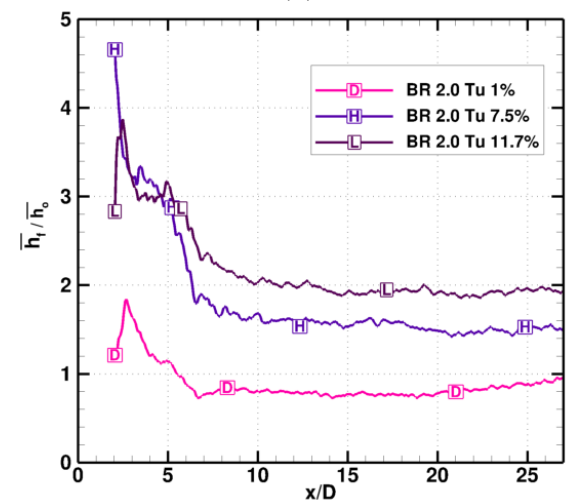

(d)

Fig. 12. Span-Averaged Heat Transfer Coefficient for Constant Blowing Ratio and Varying Turbulence Intensity

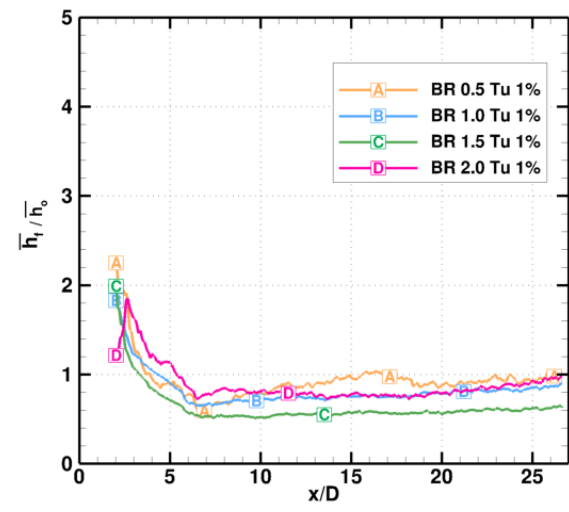

(a)

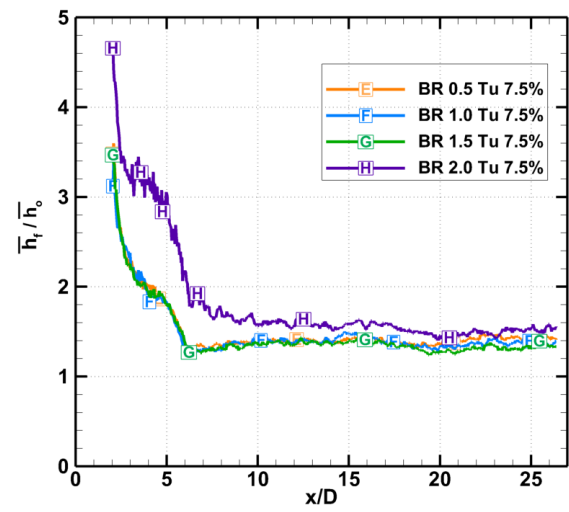

(b)

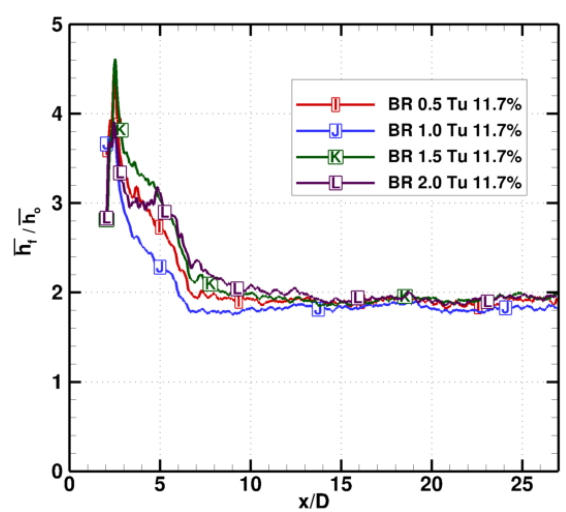

(c)

Fig. 13. Span-Averaged Heat Transfer Coefficient for Constant Turbulence Intensity and Varying Blowing Ratio 


\subsubsection{Heat Transfer Coefficient and Net Heat Flux Reduction (NHFR)}

To thoroughly analyze the performance of a cooling hole geometry the convective heat transfer coefficient must be taken into account. Heat transfer to a film cooling airfoil is defined in equation (20) where (q") is the heat flux per unit area.

$$
q^{\prime \prime}=h_{f}\left(T_{a w}-T_{w}\right)
$$

Sen et al. [21] introduced a parameter known as net heat flux reduction (NHFR) which determines the net benefit resulting from film cooling. The NHFR parameter is defined in equation (21). This term serves to quantify the relationship between the non-dimensional convective heat transfer coefficient and the adiabatic film cooling effectiveness.

\section{NHFR=1-q"qo"=hf(Taw-Tw $) h o(T \infty-T w)$}

A dimensionless temperature is defined in equation (22) and is used to reduce the NHFR equation to its most advantageous form, as presented in equation (23). The dimensionless temperature $(\theta)$ of 1.6 is used and is considered to be representative of engine conditions [21].

$\theta=T_{\infty}-T_{c} T_{\infty}-T_{w}=1.60$

NHFR $=1-$ hfho $(1-$ naw $\theta)$

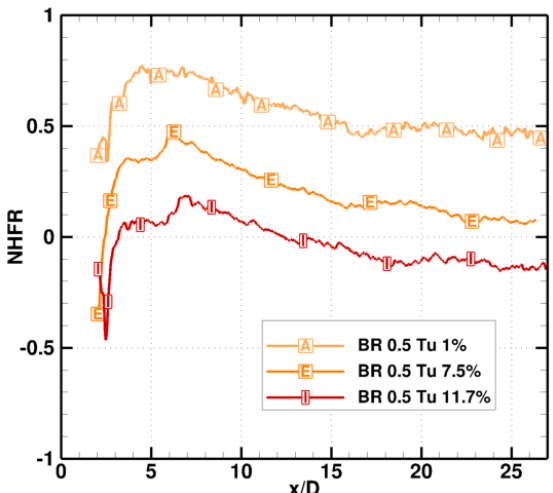

(a)

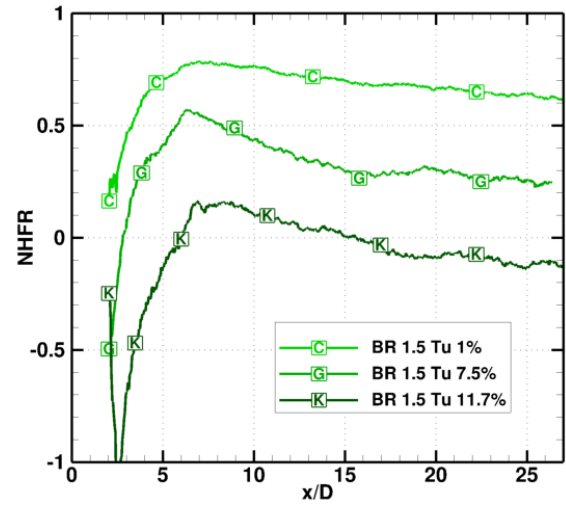

(c)
The NHFR can be negative in areas where the film cooling effectiveness is especially high and the non-dimensional convective heat transfer coefficient is also high, such as the region directly at the trailing edge of the coolant hole $(x / D=2)$. The typical range however for NHFR is typically between zero (0) to one (1). The NHFR data will be presented along with span-averaged heat transfer coefficient data.

The dimensionless span-averaged heat transfer coefficient is presented in Fig. 12 at a constant blowing ratio for varying freestream turbulence intensity. Fig. 13 shows the dimensionless span-averaged heat transfer coefficient for varying blowing ratio at constant freestream turbulence.

At freestream turbulence intensities of $7.5 \%$ and $11.7 \%$, the heat transfer coefficient is increased as the blowing ratio increases. This is expected because as the freestream moves around the coolant jets, which primarily occurs in the first 7 hole diameters downstream, the local turbulence intensity is increased as the coolant jets and freestream mix resulting in a higher heat transfer coefficient. As the freestream turbulence intensity is increased the heat transfer coefficient is increased for all cases, and this effect is particularly noticeable compared to the differences between blowing ratios at a given turbulence intensity.

The span-averaged net heat flux reduction (NHFR) is presented in Fig. 14 for constant blowing ratio and varying freestream turbulence and in Fig. 15 at constant freestream turbulence and varying freestream blowing ratio. The locations of the lowest NHFR are the locations where the heat transfer is highest, specifically at the trailing edge of the coolant hole at a non-dimensional distance $(\mathrm{x} / \mathrm{D})$ of 2 . The best overall performing case based on the NHFR is the case when the blowing ratio is 1.5 and the freestream turbulence intensity is $1 \%$. The blowing ratio of 1.5 is the best performer when considering the NHFR for turbulence intensities of 1 and $7.5 \%$, but for the $11.7 \%$ freestream turbulence intensity cases, a blowing ratio of 1.0

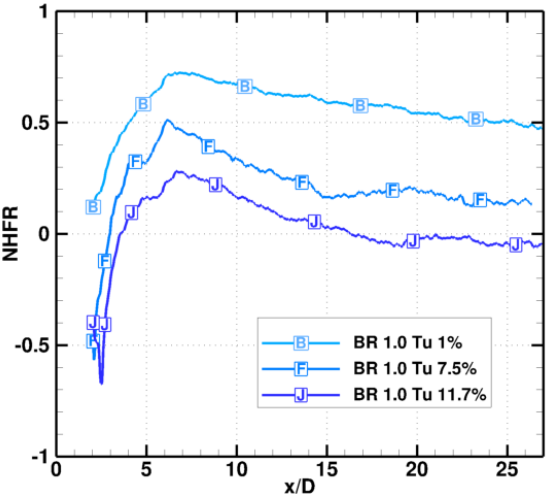

(b)

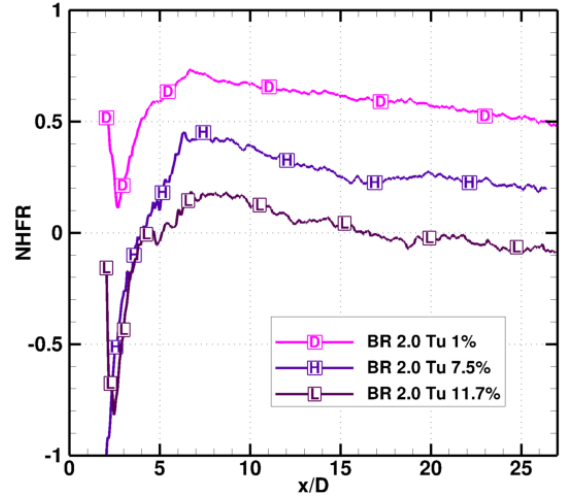

(d) 
Fig. 14. Span-Averaged NHFR for Varying Turbulence Intensity and Constant Blowing Ratio 


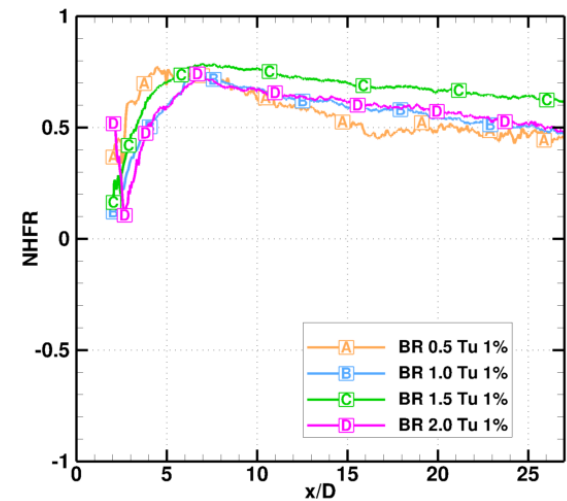

(a)

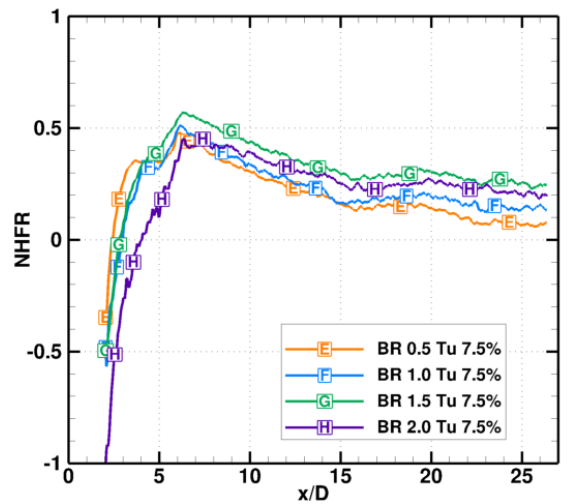

(b)

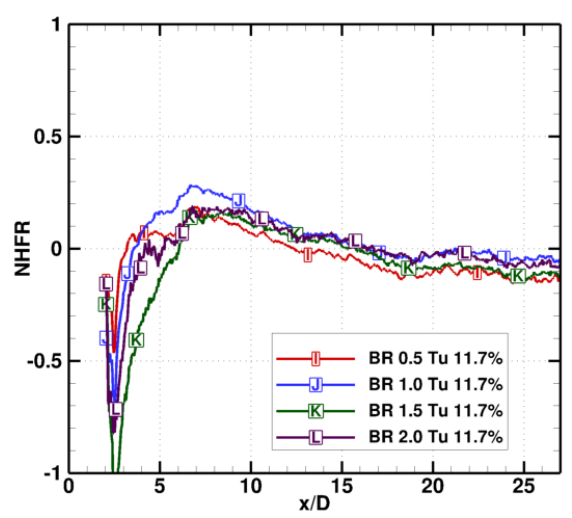

(c)

Fig. 15. Span-Averaged NHFR for Constant Turbulence Intensity and Varying Blowing Ratio

was found to be the best blowing ratio. The peak for all cases appears to occur between 5-7 hole diameters downstream or the main hole.

The area-averaged dimensionless heat transfer coefficient is beneficial because general ideas about the heat transfer coefficient may be presented to show how varying blowing ratio and freestream turbulence intensity can change the overall amount of heat transfer. Table 4 presents the areaaveraged convective heat transfer coefficient, and as expected the heat transfer coefficient is increased in the presence of increased turbulence. The interesting trend to notice is that for the two lower turbulence cases the heat transfer is lowest for a blowing ratio of 1.5 , while at the highest freestream turbulence intensity, the lowest heat transfer occurred at a blowing ratio of 1.0 .

\section{Table 4}

Area-averaged Dimensionless Heat Transfer Coefficient

\begin{tabular}{cccc}
\hline Case & Tu 1.00\% & Tu 7.50\% & Tu 11.7\% \\
\hline BR 0.50 & 0.929 & 1.506 & 2.103 \\
\hline BR 1.00 & 0.820 & 1.483 & 1.964 \\
\hline BR 1.50 & 0.632 & 1.456 & 2.175 \\
\hline BR 2.00 & 0.886 & 1.821 & 2.172 \\
\hline
\end{tabular}

\section{Conclusions}

This facility is the first to experimentally investigate the impact of elevated freestream turbulence intensity and the effects on the performance of an anti-vortex hole (AVH) geometry. The primary concern with the geometry is the influence elevated freestream turbulence intensity will have on the effectiveness of the secondary holes mitigating the counter rotating vortex (CRV) pair of the main coolant hole. The AVH design used in this study had previously been investigated by Dhungel et al. [8,15], Heidmann, Ekkad et al. [6,7], Hunley et al. [9], and Repko et al. [10,11,12] and showed that there was a considerable improvement of film cooling effectiveness over the straight hole geometry. Hunley et al. [9] and Repko et al. [10,11,12] studied the effect of turbulence intensities of 5, 10, and $20 \%$ for a blowing ratio of 2.0 and found that elevated levels of freestream turbulence predicted improvements in the span-averaged film cooling effectiveness. The highest turbulence case was found to be the best performer when looking at the span-averaged film cooling effectiveness, which shows indications that the
CRV pairs from the secondary holes are not interfered with by the freestream turbulence. This prediction is contradictory to the current experimental investigation. In this study it was found that the best performing case based on the area-averaged film cooling effectiveness was the blowing ratio of 2.0 and turbulence intensity of $7.5 \%$ case. At this intermediate turbulence intensity the coolant jets are still diffused laterally and the secondary holes appear to be much more effective than in the $11.7 \%$ turbulence cases. The $11.7 \%$ cases, although outperformed by the $7.5 \%$ cases, still performed better than the $1 \%$ cases. This shows that elevated freestream turbulence intensity increases the film cooling effectiveness of the AVH, but the $11.7 \%$ cases experienced a lower film cooling effectiveness than the $7.5 \%$ cases indicating that freestream turbulence intensities greater than $11.7 \%$ could cause performance issues. The highest local film cooling effectiveness occurs directly at the trailing edge of the coolant hole and again 5 cooling hole diameters downstream. It can be ascertained from this study that at high blowing ratios, which are more realistic to engine conditions, the film cooling effectiveness both spanaveraged and on the centerline is enhanced by high freestream turbulence.

The convective heat transfer coefficient is increased when the freestream turbulence intensity is increased and also when the blowing ratio is increased. The best overall performing case based on the net heat flux reduction is the case when the blowing ratio is 1.5 and the freestream turbulence intensity is $1 \%$. The blowing ratio of 1.5 is the best performer when considering the NHFR for turbulence intensities of 1 and $7.5 \%$, but for the freestream turbulence intensity cases, a blowing ratio of 1.0 was found to be the best performing blowing ratio.

The AVH design studied is practical in its approach and could certainly be employed in real gas turbine applications. This design could be much easier to manufacture than that of current shaped holes and would allow for less air to be extracted from upstream in the compressor section leading to higher engine efficiencies. Furthermore, the longevity of the components could be increased reducing the required replacement of components and engine downtime. The only issue with the production of the current design would be the manufacture of the smaller secondary holes and as such current research is being performed by LeBlanc et al. [22] to implement secondary holes with a diameter similar to the main hole.

\section{Acknowledgements}

The authors would like to acknowledge the support of the Department of Energy, Office of Science, Experimental Program to Stimulate Competitive 
Research (EPSCoR) under grant/contract number DE-FG02-09ER46615, monitored by Dr. Tim Fitzsimmons of the DOE Office of Science/EPSCoR. The authors would also like to thank the members of the West Virginia University Center for Alternative Fuels Engines and Emissions (WVU CAFEE) as well as the WVU Statler College and Mechanical and Aerospace Engineering department for their support throughout the program.

\section{References}

[1] Kadotani, K. and Goldstein, R., "On The Nature of Jets Entering A Turbulent Flow: Part A-Jet-Mainstream Interaction," Journal of Engineering for Gas Turbines and Power, vol. 101, pp. 459-465, 1979.

[2] Kadotani, K. and Goldstein, R. "On the Nature of Jets Entering A Turbulent Flow: Part B-Film Cooling Performance," Journal of Engineering for Gas Turbines and Power, vol. 101, pp. 466-470, 1979.

[3] Mehendale, A.B. and Han, J.C., "Flat Plate Film Cooling With Steam Injection Through One Row and Two Rows of Inclined Holes," ASME Journal of Turbomachinery, vol. 108, pp. 595-607, 1986.

[4] Haven, B.A., Yamagata, D.K., Kurosaka, M., Yamawaki, S., and Maya, T., "Anti-Kidney Pair of Vortices in Shaped Holes and Their Influence on Film Cooling Effectiveness," ASME Paper 97-GT-451997.

[5] Bunker, R.S., "A Review of Shaped Hole Turbine Film-Cooling Technology," Journal of Heat Transfer, vol. 127, pp. 441-453, 2005.

[6] Heidmann, J.D. and Ekkad, S.V., "A Novel Anti-Vortex Turbine Film Cooling Hole Concept," ASME Paper GT2007-27528.

[7] Heidmann, J.D., "A Numerical Study of Anti-Vortex Film Cooling Designs at High Blowing Ratio," ASME Paper GT2008-50845.

[8] Dhungel, A., Lu, Y., Phillips, W., Ekkad, S.V., and Heidmann, J.D., "Film Cooling From a Row of Holes Supplemented with Anti Vortex Holes," ASME Paper GT2007-27419.

[9] Hunley, B. K., Nix, A.C., and Heidmann, J.D., "A Preliminary Numerical Study on the Effect of High Freestream Turbulence on AntiVortex Film Cooling Design at High Blowing Ratio," ASME Paper GT2010-22077.

[10] Repko, T.W., Nix, A.C., and J. D. Heidmann, "A Parametric Numerical Study of the Effects of Freestream Turbulence Intensity and Length Scale on Anti-Vortex Film Cooling Design at High Blowing Ratio," ASME paper HT2013-17255.

[11] Repko, T.W., "Numerical Investigation of the Influence of Elevated Turbulence Levels on the Cooling Effectiveness of an Anti-Vortex Hole Geometry," Master of Science in Aerospace Engineering, Mechanical and Aerospace Engineering, West Virginia University, 2014.

[12] Repko, T.W., Nix, A.C., Uysal S.C. and Sisler, A.T, "Flow Visualization of Multi- Hole Film-Cooling Flow under Varying Freestream Turbulence Levels," Journal of Flow Control, Measurement \& Visualization, 4, 13-29.

[13] Bradshaw, P. and Mehta, R. (2014, Sep. 29). Wind Tunnel Design. Available: http://navier.stanford.edu/bradshaw/tunnel/index.html

[14] Lu, Y., "Effect of Hole Configurations on Film Cooling from Cylindrical Inclined Holes for the Application to Gas Turbine Blades," Ph. D thesis, Virginia Polytechnic Institute and State University, 2007.

[15] Dhungel, A., "Film Cooling from a Row of Holes Supplemented with Anti Vortex Holes" Master Thesis, Louisiana State University 2007

[16] Ekkad, S.V., Ou, S., Rivir, R.B., "A Transient Infrared Thermography Method for Simultaneous Film Cooling Effectiveness and Heat Transfer Coefficient Measurements From a Single Test," ASME Journal of Turbomachinery, vol. 126, pp. 597-603, October 2004.
[17] Vendula, R.J. and Metzger, D.E., "A Method for the Simultaneous Determination of Local Effectiveness and Heat Transfer Distributions in Three-Temperature Convection Situations," ASME Paper 91-GT-345.

[18] Moffat, R.J., "Describing the uncertainties in experimental results," Experimental Thermal and Fluid Science, vol. 1, pp. 3-17, 1988.

[19] Bons, J. P., MacArthur, C. D., and Rivir, R., "The Effect of High Freestream Turbulence on Film Cooling Effectiveness," ASME Journal of Turbomachinery, vol. 118, pp. 814-825, 1994.

[20] Kohli, A., Bunker, D.G., "Adiabatic Effectiveness, Thermal Fields, and Velocity Fields for Film Cooling With Large Angle Injection," ASME Journal of Turbomachinery, vol. 119, pp. 352-358, April 1997.

[21] Sen, B., Schmidt, D.L., and Bogard, D.G., "Film cooling with compound angle holes: heat transfer," ASME Journal of Turbomachinery, vol. 118, pp. 800-806, 1996.

[22] LeBlanc, C., Narzary, D.P., Ekkad, S.V., "Film-Cooling Performance of Antivortex Hole on a Flat Plate," ASME Journal of Turbomachinery, vol. 135, November 2013. 